ArtefaCToS. Revista de estudios de la ciencia y la tecnología

eISSN: 1989-3612

Vol. 10, No. 1 (2021), 2. ${ }^{\text {a }}$ Época, 5-10

DOI: https://doi.org/10.14201/art2021101510

Introducción a la sección monográfica

\title{
Ronald N. Giere, un referente y maestro de la filosofía de la ciencia
}

Introduction to monographic section

Introduction: Ronald N. Giere, A Reference and Mentor of Philosophy of Science

\author{
Anna ESTANY*; Ana CUEVAS BADALLO** \\ ${ }^{*}$ Editora invitada/Guest editor \\ Universitat Autònoma de Barcelona, España \\ anna.estany@uab.cat \\ ** Universidad de Salamanca, España \\ acuevas@usal.es
}

Ronald N. Giere (1938-2020) nació en Cleveland, Ohio, Estados Unidos. Estudió física, doctorándose en Cornell University en Filosofía en 1968. En su larga carrera académica fue profesor en New York University y en University of Pittsburgh. Estuvo también en el Departamento de Historia y Filosofía de la Ciencia de Indiana University (Bloomington) y posteriormente en University of Minnesota. Pertenecía a la Asociación Estadounidense para el Avance de la Ciencia, fue miembro del consejo editorial de la revista Philosophy of Science y antiguo presidente de la Philosophy of Science Association. Ha sido uno de los referentes de la filosofía de la ciencia de nuestra época, con un impacto muy importante a nivel internacional.

El objetivo del monográfico en ArtefaCToS como homenaje a la figura de Ronald N. Giere es poner de manifiesto su pensamiento en las cuestiones claves que ha abordado la filosofía de la ciencia a lo largo de buena parte del siglo XX y que continúa siendo un punto de referencia para los retos que la filosofía tiene planteados actualmente. Vamos a señalar algunas de sus obras más significativas y sus ideas desarrolladas en las mismas.

En Espańa su obra ha tenido muy buena acogida, siendo un referente no solo para la comunidad de la filosofía de la ciencia, sino también en otros campos de 
conocimiento como la didáctica de las ciencias. Una muestra de su interés por la enseñanza es su libro Understanding Scientific Reasoning (1979), donde introduce al estudiante a razonar críticamente mediante estudios de caso, tanto históricos como actuales, para desarrollar su competencia científica y tecnológica.

La estancia de Giere en el Departamento de Historia y Filosofía de la Ciencia en Indiana University enlaza con el análisis de la relación entre historia y filosofía de la ciencia, una de las cuestiones que abordó en el artículo de referencia sobre esta cuestión "History and philosophy of science: Intimate relationship or marriage of convenience?" (1973).

En Science without laws (1999) Giere afronta el debate sobre si el conocimiento científico es objetivo e inapelable o puede estar implicado por factores sociales, y también si las verdades científicas son independientes de los valores y creencias humanas. Así ofrece una perspectiva mediadora entre verdades universales y relativismo, argumentando que es mejor comprender que los científicos simplemente elaboran modelos más o menos abstractos de concepciones limitadas del mundo.

Otra de las orientaciones de Giere tuvo como escenario el debate sobre realismo vs. instrumentalismo de las teorías científicas, que desarrolló en Scientific Perspectivism (2006). La idea básica que allí desarrolla es que las teorías nos proporcionan diferentes facetas de la realidad sobre el conocimiento del mundo, a diferencia del instrumentalismo que considera las teorías como simples herramientas conceptuales para abordar la realidad, ya que no tenemos justificación epistémica de que las reflejen.

Giere fue uno de los más importantes impulsores del enfoque cognitivo de la filosofía de la ciencia con su obra seminal Explaining Science: A Cognitive Approach (1988). Este enfoque forma parte del programa naturalizador en filosofía, en el sentido de que los principios epistémicos no pueden hacer caso omiso de los resultados de las ciencias empíricas, cuestionando así el modelo apriorista de la fundamentación del conocimiento. Dentro del trabajo de Giere en el marco del enfoque cognitivo hay que destacar la edición del volumen Cognitive models of science (1992), fruto de unas jornadas en las que, según sostenía en la Introducción, la inteligencia artificial, la psicología cognitiva y la neurociencia cognitiva, han alcanzado la madurez suficiente para que sean ahora un recurso valioso para los filósofos de la ciencia.

El pensamiento de Giere se enmarca en la "concepción semántica de las teorías", como alternativa a la "concepción sintáctica" inspirada en el Círculo de Viena y centrada en las teorías científicas que pueden ser verdaderas o falsas en función de que todos sus enunciados sean verdaderos o haya alguno falso. La concepción semántica pone en el centro de la representación del conocimiento la idea de "modelo teórico", una entidad mental no lingüística con la que los humanos representamos el conocimiento. 
En la exposición de la obra de Giere no podemos olvidar una serie de artículos de las dos últimas décadas sobre la cognición distribuida y la relación entre lo cognitivo y lo social, tales como "Distributed cognition in epistemic cultures" (2002), "Scientific cognition as distributed cognition" (2002), "Distributed Cognition: Where the Cognitive and the Social Merge" (2003), entre otros. Forman parte de su aproximación a la ciencia de forma integral en el sentido de tener en cuenta todos los factores que intervienen en la práctica científica. Giere considera que la actividad científica, particularmente en las ciencias experimentales, involucra la operación de sistemas cognitivos distribuidos, tal como se entiende en las ciencias cognitivas contemporáneas.

No cabe duda de que el pensamiento de Ronald N. Giere fue innovador, trazando puentes entre teorías filosóficas que parecían incompatibles, situando en la agenda de la filosofía de la ciencia su aplicabilidad a la enseñanza de las ciencias, a la tecnología, a la relación entre historia y filosofía de la ciencia e integrando los factores sociales en la práctica científica. Todo ello en el marco interdisciplinar de las ciencias cognitivas.

Los autores que han sido seleccionados para formar este número monográfico han querido rendir un homenaje a este gran filosofo de la ciencia, analizando algunos de los temas sobre los que Giere realizó una aportación personal y original.

En primer lugar, David Casacuberta y Anna Estany, profesores de la Universitat Autònoma de Barcelona, España, contribuyen a este número con su artículo sobre: "La cognición distribuida en el pensamiento de Ronald Giere". En él se analiza la relevancia que tuvo en su filosofía de la ciencia la idea de cognición distribuida del antropólogo cognitivo Edwin. A partir de esta, Giere construyó una explicación acerca de lo que es la cultura epistémica, la cognición y la mente, la agencia, la relación entre lo cognitivo y lo social y cómo los nuevos recursos computacionales pueden influir en el modo de hacer ciencia. Por ejemplo, desarrolló una investigación sumamente interesante sobre cómo se desarrolla la cognición científica los laboratorios, algo que podría entenderse mejor si se partía precisamente de la idea de que la cognición se produce de manera colectiva, a través de esa cognición distribuida.

A continuación, Francesco Consiglio y Fernando Martínez Manrique, de la Universidad de Granada, España, también ahondan en la noción de cognición distribuida en Giere, en su artículo "Cognición distribuida: entre lo individual y lo social". Allí, los autores explican el origen de la noción de cognición distribuida en los ejemplos de la navegación y del procesamiento distribuido en paralelo, así como la aplicación que Giere hace al caso de la ciencia. Analizan cómo esta noción tiene ventajas explicativas dentro de su perspectivismo, y cómo se puede llegar a eliminar los límites entre lo cognitivo y lo social. Consiglio y Martínez también analizan la propuesta de Giere en relación con los debates sobre la mente extendida, mostrando algunas críticas que ellos consideran que no interpretan bien su posición. Para finalizar, reevalúan la reticencia que Giere mostró a la 
extensión de ciertas propiedades mentales a los sistemas distribuidos, presentando dos intentos recientes de establecer las condiciones en que esta extensión es razonable.

Antonio Diéguez, profesor de la Universidad de Málaga, España, presenta en su artículo "El naturalismo de Ronald Giere", la estrategia naturalista que este adoptó y que provenía fundamentalmente del uso de las herramientas que nos proporcionan las ciencias cognitivas (psicología cognitiva, lógica, inteligencia artificial, neurociencias, lingüística, filosofía de la mente, etc.) y también, aunque menos, de las ciencias sociales. Según Diéguez, Giere, superó con su propuesta una concepción filosófica que veía a la ciencia como el resultado de una racionalidad categórica e incondicionada, guiada por la lógica formal, y descontextualizada histórica y socialmente. Sin embargo, Diéguez considera que la alternativa que Giere escoge también tiene sus problemas, ya que también termina sosteniendo una visión limitada de la racionalidad. La racionalidad condicionada significa, en Giere, que los principios de racionalidad admisibles son solo principios que conectan las estrategias de investigación con los fines de la investigación. Es una racionalidad instrumental a la que se quiere subordinar cualquier consideración sobre los fines.

Por ello, pese al interés de su propuesta, la pretensión de Giere de hacer de la epistemología una rama de la psicología o de la biología puede considerarse una reacción excesiva frente a la epistemología tradicional y del logicismo de los neopositivistas.

Javier Echeverria, investigador en Jakiunde, España, a su vez, analiza también el perspectivismo de Giere, exponiendo que, en sus reflexiones epistemológicas se incluyen disimuladamente ciertas afirmaciones valorativas, sobre las cuales Giere no reflexiona. Según Echeverria, esto es una insuficiencia significativa, sobre todo porque Giere analiza con detalle algunos ejemplos relevantes de la tecnociencia contemporánea. Al hilo del discurso de Giere, Echeverría propone un perspectivismo axiológico en filosofía de la ciencia, pero sobre todo en filosofía de la tecnociencia. En el texto, Echeverría considera que las posturas de Giere son más matizadas y sofisticadas que las de la mayoría de los epistemólogos actuales, pero a pesar de ello, cuando llega el momento de ilustrar su perspectivismo epistemológico (que no científico) recurre a los procesos cognitivos, y no a los procesos interactivos en general, pese a que en algún momento subraya la importancia del concepto "interacción", mucho más amplio y fundamental que el de la cognición, según Echeverría, al menos en el caso de las tecnociencias.

Mario Gensollen de la Universidad Autónoma de Aguascalientes, México y Marc Jiménez-Rollan Universidad Autónoma Metropolitana (Unidad Cuajimalpa) también en México exploran en "Giere’s scientific perspectivism as carte blanche realism" las contribuciones de Giere al debate sobre el realismo científico. Consideran que estas tienen mucho que ver con su perspectivismo, y defienden que esa concepción de Giere es una forma de realismo carte blache, es decir: 
un concepto de acuerdo con el cual la ciencia tiene como objetivo la verdad, y cuenta con elementos para justificar que la ha obtenido. Sin embargo, no lo hace depender de algún rasgo específico de las representaciones científicas.

Mercè Izquierdo Aymeric de la Universitat Autònoma de Barcelona, España, y Agustín Adúriz-Bravo de la Universidad de Buenos Aires, Argentina, participan en este monográfico con un articulo con el título "Contribuciones de Giere a la reflexión sobre la educación Científica”. En él muestran cómo Giere, en su larga carrera como filósofo, se interesó por reflexionar sobre la enseñanza de las disciplinas científicas con el auxilio de ideas tales como: reconstrucción modelo-teórica, realismo perspectivo, modelos como mapas, hipótesis teóricas y didáctica basada en el agente (cognitivo). Esas reflexiones son de gran utilidad para el profesorado a la hora de diseñar una enseñanza de las ciencias en los distintos niveles educativos que procure pensamiento autónomo y crítico en el estudiantado. El análisis naturalista de Giere, que expone el trabajo de los científicos tal como se realiza en los laboratorios, puede ser transpuesto para caracterizar la ciencia escolar como una forma justificada y autocorrectiva de aprendizaje.

Rodrigo López-Orellana de la Universidad de Valparaíso, Chile y Alger Sans Pinillos de la Università degli Studi di Pavia, Italia, aportan su artículo acerca de "Ronald Giere, ¿semanticista? Una pregunta provocativa para el debate contemporáneo sobre la representación científica". En él señalan que puede ser mejor considerar el enfoque de Ronald Giere separado de lo que se ha llamado la familia semanticista en filosofía de la ciencia. El argumento que emplean para defender esta idea es que Giere rechaza las relaciones diádicas de representación isomórfica y de verdad para la representación científica típicas de la concepción semanticista. De hecho, López Orellana y Sans afirman que la filosofía de Giere encaja mejor en una interpretación pragmática, que a su vez permitiría también caracterizar sus ideas acerca de la práctica científica con modelos, así como con el debate contemporáneo sobre la representación.

Y, por último, Ana Cuevas y Obdulia Torres de la Universidad de Salamanca, España, en "Las ciencias sociales e ingenieriles desde el realismo constructivo de Ronald Giere" analizan cómo el realismo constructivo defendido por Giere puede ser de utilidad para una mejor caracterización de dos ámbitos de la investigación científica que tienden a ser menospreciados por las concepciones filosóficas más tradicionales: el conocimiento social y el ingenieril. En el artículo defienden que esos dos ámbitos comparten rasgos relevantes y que la propuesta modelista del autor permite dar mejor cuenta de sus peculiaridades. Para ello emplean la noción de similitud que los modelos guardan con el mundo, y cómo esta postura incide en la elección entre teorías. La naturaleza especial de las leyes en ciencias sociales y en las ciencias ingenieriles, así como los criterios por los que los científicos en estos ámbitos eligen entre los distintos modelos posibles se entienden mejor dentro de la concepción de Giere frente a las concepciones más clásicas sobre las teorías científicas. 
Además, hemos incluido una sección de Evocaciones personales en las que dos de sus estudiantes, Eric Christian Barnes y Gurol Irzik, recuerdan a su maestro y cómo este les influyó tanto en el plano académico como personal, mostrando la generosidad que Giere siempre tuvo como profesor y como investigador.

Giere ha sido un filósofo de la ciencia que nos ha ayudado a tener una visión de esta mucho más compleja y cercana al trabajo de los propios investigadores. Todos los autores que contribuimos a este monográfico queremos mostrarle nuestro agradeciendo y homenaje con este monográfico. 